\section{Stimulus-Bound Behavior}

Chava Creque $^{1}$ and Stephanie A. KolakowskyHayner $^{2}$

${ }^{1}$ Department of Psychology and Neuroscience, University of Colorado Boulder, Boulder, CO, USA

${ }^{2}$ Icahn School of Medicine at Mount Sinai, New York, NY, USA

\section{Synonyms}

Automatic behavior; Stimulus-driven behavior

\section{Definition}

Stimulus-bound behavior is commonly found in frontal lobe syndrome and other executive functioning disorders and is a response to a specific stimulus in one's environment - an externally oriented cognitive approach. For example, the behavior displayed seems to depend primarily upon available objects and subject predisposition, rather than the activation of a specific drive such as hunger, anger, sex drive, etc. A person exhibiting stimulus-bound behavior may feel compelled to use certain items present, regardless of an external need or any instruction to do so. When a stimulus is encountered, the person may act without thinking or considering other factors.
The behaviors are often perseverative in nature and focus on partial information. Immediate stimulus-bound behavior often does not take into account future consequences or long-term outcomes and causes difficulty with planning, organizing, and behavioral initiative.

\section{Cross-References}

- Executive Functioning

- Frontal Lobe Syndrome

Frontal Lobes

\section{References and Readings}

Archibald, S., Mateer, C., \& Kerns, K. (2001). Utilization behavior: Clinical manifestations and neurological mechanisms. Neuropsychology Review, 11(3), 117-130.

Feinstein, A., Levine, B., \& Protzner, A. (2000). Confabulation and multiple sclerosis: A rare association. $\mathrm{Mul}$ tiple Sclerosis, 6(3), 186-191.

Jackson, A., \& Haverkamp, B. (1991). Family response to traumatic brain injury. Counselling Psychology Quarterly, 4(4), 355.

Long, C. J., \& Ross, L.K. (Eds.). (1992). Handbook of head trauma: Acute care to recovery. Springer.

Suedfeld, P. (1988). Are simple decisions always worse? Society, 25(5), 25-27.

Wachtel, E. (1976). The multiple meaning of symptoms: A case illustration. Psychotherapy: Theory, Research \& Practice, 13(3), 219-224. 